\title{
Constituintes químicos fixos e voláteis dos talos e frutos de Piper tuberculatum Jacq. e das raízes de $P$. hispidum H. B. K.
}

\author{
Valdir Alves FACUNDO ${ }^{1}$, Aline Roberta POLLLI ${ }^{2}$, Rosely Valéria RODRIGUES ${ }^{3}$, \\ Júlio S. L Teixeira MILITÃO ${ }^{4}$, Rodrigo guerino STABELLI ${ }^{5}$, Cosuelo Tamiris CARDOSO ${ }^{6}$
}

\section{RESUMO}

Os óleos essenciais dos frutos e talos finos de Piper tuberculatum e das raízes de P. hispidum, coletados no estado de Rondônia, foram obtidos por hidrodestilação e analisados por GC e GC-MS. Foram identificados como constituintes majoritários, nos óleos dos frutos e talos finos de $P$. tuberculatum, o óxido de cariofileno (32,1\%) e (26,6\%) e o $(E)$-cariofileno $(17,7 \%)$ e $(12,3 \%)$, respectivamente. No óleo essencial das raízes de P. hispidum, foram identificados, como constituintes majoritários, o dilapiol (57,5\%), a elemicina $(24,5 \%)$ e o apiol (10,2\%). Do extrato etanólico dos frutos de P. tuberculatum, foram isolados os esteróides $\beta$-sitosterol e estigmasterol, as amidas piplartina e dihidropiplartina e um derivado do ácido cinâmico, o ácido 3,4,5-trimetoxi-dihidrocinâmico.

PALAVRAS-CHAVE: Piper tuberculatum, Piper hispidum, óleo essencial, amidas.

\section{Fixed and volatile chemical constituents from stems and fruits of Piper tuberculatum Jacq. and from roots of $P$. hispidum H. B. K.}

\begin{abstract}
The essential oils of the fruits and fine stems of Piper tuberculatum and of the roots of P. hispidum, collected in the state of Rondônia, had been gotten by hydrodistillation and analyzed by GC and GC-MS. Caryophyllene oxide - 32,1\% in fruits and $26,6 \%$ in fine stem, and $(E)$-caryophyllene $-17,7 \%$ in fruits and 12,3\% in fine stems, were identified as the major constituents in such parts of $P$. tuberculatum. In the essential oil of the roots of $P$. hispidum, dillapiol (57,5\%), elemicine (24,5\%) and apiole $(10,2 \%)$ were identified as the most abundant constituents. From the ethanolic extract of the fruits of $P$. tuberculatum, the steroids $\beta$-sitosterol and stigmasterol, the amides piplartine and dihidropiplartine and the derivative of the cinâmico acid 3,4,5-trimethoxy-dihidrocinâmic acid were isolated.
\end{abstract}

KEY WORDS: Piperaceae, Piper tuberculatum, Piper hispidum, essential oil, amides.

\footnotetext{
1 Universidade Federal de Rondônia, vfacundo@unir.br

2 Universidade Federal de Rondônia, xpolli@hotmail.com

3 Universidade Federal de Rondônia, rvaleria@unir.br

${ }^{4}$ Universidade Federal de Rondônia, militao@unir.br

5 Universidade Federal de Rondônia, stabelli@unir.br

${ }^{6}$ Universidade Federal de Rondônia, consutamiris@gmail.com
} 


\section{INTRODUÇÃO}

O gênero Piper pertencente à família Piperaceae, encontrase distribuído nas regiōes tropicais e subtropicais de todo mundo. Muitas espécies de Piper são usadas para fins curativos em diversas culturas (Bezerra et al., 2007). O histórico do gênero Piper descrito por Parmar et al. (1997), relata o uso de espécies para o tratamento de algumas enfermidades em diferentes povos. Na China, algumas prescrições recomendam o uso das folhas de $P$. futokasura no tratamento de arritmias cardíacas e da asma. Na Jamaica dores estomacais são tratadas com uma infusão das folhas de $P$. aduncum e $P$. hispidum. No México e no Brasil, usa-se as folhas de $P$. amalago para aliviar dores estomacais e no combate a diversas infecções. Folhas e talos de $P$. marginatum e $P$. tuberculatum são utilizadas, na Paraíba, contra picada de cobra e como sedativos (Chaves et al., 2006; Araújo-Junior et al., 1999). Silva et al. (2007) demonstraram que os extratos das folhas e raízes de $P$. aduncum, uma planta medicinal, apresentaram atividades inseticida sobre adultos de Aetalion sp (cigarrinha). Os óleos essenciais das partes aéreas de plantas dessa espécie, coletadas em diferentes localidades da regiāo Amazônica, apresentaram grandes concentraçôes do fenilpropanóide dilapiol, o qual foi considerado o responsável pelo efeito inseticida relatado por Maia et al. (1998). Outra espécie de relevante valor comercial é a $P$. hispidinervum por apresentar um óleo essencial rico em safrol, um fenilpropanóide, muito utilizado nas industrias de cosméticos e inseticidas (Bergo et al., 2005). Recentemente, foi divulgada a atividade larvicida, contra o Aedes aegypti, do óleo essencial de quatro espécies de Piper da região Amazônica, $P$. gaudichaudianum, $P$. permucronatum, $P$. humaytanum e $P$. hostmanianum (Moraes et al., 2007).

P. tuberculatum Jacq e P. hispidum H. B. K, são conhecidas como pimenta d'ardo e jaborandi ou falso-jaborandi, respectivamente, (Araújo-Junior et al., 1999; Albiero et al., 2006). A distribuição geográfica destas duas espécies se estende pelas Américas, do México à Argentina. No Brasil elas ocorrem nos estados do Amazonas, Pará, Piauí, Ceará, Paraíba, Pernambuco, Bahia, Rio de Janeiro, Paraná, Santa Catarina, Mato Grosso, São Paulo e Mato Grosso do Sul (Guimarães e Giordano, 2004).

Os estudos da constituição química dos óleos essenciais de P. tuberculatum e P. hispidum têm revelado que os resultados nem sempre são uniformes (Machado et al.,1994; Cysne et al., 2005; Facundo et al., 2005a; Mesquita et al., 2005; Navickiene et al., 2006; Potzernheim et al., 2006). Isto pode ser atribuído à diversidade genética das espécies, ocorrendo uma variedade de quimiotipos. Outros fatores que também podem ser considerados são: a idade foliar da planta, as variáveis ambientais e as metodologias utilizadas pelos diferentes autores.
Entre os constituintes fixos isolados de $P$. hispidum e $P$. tuberculatum destacam-se as amidas do tipo isobutílicas, pirrolidínicas, dihidropiridonas, piperidinas, derivados prenilados do acido benzóico, derivados do ácido cinâmico e flavonóides (Parmar et al. 1997).

Neste trabalho relata-se a composição química dos óleos essenciais obtidos dos frutos e talos finos de P. tuberculatum e das raízes de $P$. hispidum, bem como, o isolamento de uma mistura de dois esteróides, 1 e 2, duas amidas, 3 e 4, e um derivado do acido cinâmico, 5 , dos frutos de $P$. tuberculatum.

\section{MATERIAL E MÉTODOS}

\section{COLETA DO MATERIAL}

As amostras de $P$. tuberculatum e $P$. hispidum foram coletadas em abril de 2005 no campus da Universidade Federal de Rondônia. A identificação botânica das plantas foi realizada pelo Dr. José Gomes do herbário do Instituto Nacional de Pesquisa da Amazônia (INPA), onde excicatas encontram-se depositadas sob os números 211724, para $P$. tuberculatum e 216630, para P. hispidum.

\section{EXTRAÇÃO DOS ÓLEOS ESSENCIAIS}

Os frutos $(1,0 \mathrm{~kg})$ e talos finos $(1,8 \mathrm{~kg})$ frescos de $P$. tuberculatum, e raízes $(2,7 \mathrm{~kg})$ frescas de $P$. hispidum, devidamente triturados foram submetidos a hidrodestilação por 4 horas, utilizando-se extratores de vidro tipo Clevenger modificado. Após a obtenção dos óleos essenciais dos frutos $(1,4 \mathrm{~mL})$, talos finos $(1,2 \mathrm{~mL})$ de $P$. tuberculatum e das raízes $(1,0 \mathrm{~mL})$ de P. hispidum, foram tratados com sulfato de sódio anidrido para a eliminação de água.

\section{ANÁLISE DOS ÓLEOS ESSENCIAIS}

Os óleos essenciais foram analisados usando um aparelho Hewlett-Packard modelo 5890 A e um instrumento GC/ MS, modelo 5973, equipado com coluna capilar (30 m x $0,25 \mathrm{~mm})$ dimetilpolisiloxano DB-5 (J\&W) $(25 \mathrm{~m} \times 0,20$ $\mathrm{mm}, 0,20 \mu \mathrm{m}$ ); gás de arraste: $\mathrm{He}$ (fluxo de $1,0 \mathrm{~mL} / \mathrm{min}$ ); as temperaturas do injetor (modelo split): $250^{\circ} \mathrm{C}$; detector de ionização de chama (FID): $270^{\circ} \mathrm{C}$; temperatura da coluna: $35-180^{\circ} \mathrm{C} / 3^{\circ} \mathrm{C} / \mathrm{min}, 180-250^{\circ} \mathrm{C} / 10{ }^{\circ} \mathrm{C} / \mathrm{min}$; $\mathrm{O}$ volume injetado $0,02 \mu \mathrm{L}$ de óleo puro. Os espectros de massas: impacto de elétrons a $70 \mathrm{eV}$. Os diversos constituintes químicos dos óleos essenciais foram identificados através dos estudos dos espectros de massas, complementados por comparação com a biblioteca do aparelho, dados da literatura e os índices retenção (Adams, 1995).

\section{ISOLAMENTOS DOS CONSTITUINTES QUIIMICOS FIXOS}

Os frutos devidamente secos e triturados de P. tuberculatum $(1,3 \mathrm{~kg})$ foram extraídos com etanol (3L x 3$)$ à temperatura 
ambiente. $\mathrm{O}$ solvente foi destilado sob pressão reduzida e forneceu $41 \mathrm{~g}$ de uma massa de coloração marrom. Parte deste material $(35 \mathrm{~g})$ foi adsorvida em sílica gel $(90 \mathrm{~g})$ e a mistura, sob a forma de pastilha, foi colocada em uma coluna cromatográfica e eluída com hexano, clorofórmio, acetato de etila e metanol. A fração obtida em clorofórmio $(9,3 \mathrm{~g})$ foi novamente submetida à cromatografia em coluna de gel de sílica e eluída com misturas de hexano e clorofórmio em gradiente de polaridade crescente, obtendo-se 83 fraçóes. As fraçôes de 10 a 19 foram reunidas, após comparação em cromatografia em camada delgada (CCD), e a fração resultante foi purificada por recristalização em clorofórmio, obtendo-se desta forma $35 \mathrm{mg}$ de um sólido branco cristalino, posteriormente identificado como sendo uma mistura de 1 e 2. As frações 32 a 54 foram reunidas, após comparação em $\mathrm{CCD}$, e a fração resultante foi submetida à cromatografia em coluna de sílica gel, eluída com hexano e acetato de etila em gradiente de polaridade crescente, obtendo-se $115,8 \mathrm{mg}$ de 3 (hexano:acetato de etila, 55:45), 21,5 mg de 4 (hexano:acetato de etila, 45:55) e 54,8 $\mathrm{mg}$ de 5 (hexano:acetato de etila, 35:65).

\section{RESULTADOS E DISCUSSÃO}

A relação dos constituintes químicos dos óleos essenciais extraídos dos talos finos e frutos de P. tuberculatum e das raízes de $P$. hispidum, suas quantidades relativas e respectivos índices de retenção (IR) estão representados na Tabela 1. Foram identificados $92,7 \%$ dos constituintes químicos detectados do óleo essencial dos talos finos de $P$. tuberculatum, dos quais $15,2 \%$ são monoterpenos e $77,5 \%$ são sesquiterpenos. Os constituintes majoritários foram o óxido de cariofileno $32,1 \%$ e o $(E)$-cariofileno $17,7 \%$. Do óleo essencial dos frutos foram identificados $90,4 \%$ dos constituintes, sendo $19,4 \%$ de monoterpenos e $71,0 \%$ de sesquiterpenos e os constituintes majoritários foram $(E)$-cariofileno com 12,3\% e o óxido de cariofileno 26,6\%. A identificação dos constituintes químicos do óleo essencial das raízes de P. hispidum foi de 99,9\%, sendo $92,2 \%$ representados por fenilpropanóides, correspondendo aos três componentes majoritários dilapiol $57,5 \%$, elemicina $24,5 \%$ e apiol $10,2 \%$.

Do extrato etanólico dos frutos de $P$. tuberculatum foram obtidos e identificados dois esteróides, o $\beta$-sitosterol 1 e o stigmasterol 2, em mistura, duas amidas, a piplartina 3 e a dihidropiplartina 4, e um derivado do acido cinâmico, o ácido 3,4,5-trimetoxi-dihidrocinâmico 5 (Figura 1).
Tabela 1 - Composição química (\%) dos talos finos e frutos de P. tuberculatum e raízes de $P$. hispidum.

\begin{tabular}{|c|c|c|c|c|}
\hline \multirow[b]{2}{*}{ Componentes } & \multirow[b]{2}{*}{$(\mathrm{IR})$} & \multicolumn{2}{|c|}{ P. tuberculatum } & \multirow{2}{*}{$\begin{array}{l}\text { P. hispidum } \\
\text { Raízes }\end{array}$} \\
\hline & & Talos finos & Frutos & \\
\hline$\alpha$-pineno & 930 & 4,4 & 4,9 & - \\
\hline canfeno & 949 & - & 0,5 & - \\
\hline verbeneno & 961 & 0,1 & 0,2 & - \\
\hline sabineno & 969 & 3,3 & 4,7 & - \\
\hline$\beta$-pineno & 975 & 0,1 & 0,1 & - \\
\hline$\alpha$-felandreno & 1003 & - & - & 2,8 \\
\hline p-cimeno & 1025 & - & - & 0,9 \\
\hline limoneno & 1028 & 2,6 & 3,6 & 1,3 \\
\hline canfonelal & 1123 & 1,5 & 0,6 & - \\
\hline trans-pinocarveol & 1140 & 1,7 & 0,9 & - \\
\hline pinocarvona & 1158 & 0,2 & 0,2 & - \\
\hline mirtenal & 1190 & 0,5 & 1,7 & - \\
\hline mirtenol & 1194 & 0,2 & 1,3 & - \\
\hline verbenona & 1200 & 0,6 & 0,5 & - \\
\hline carvona & 1250 & - & 0,2 & - \\
\hline Monoterpenos & & 15,2 & 19,4 & 5,0 \\
\hline$\alpha$-cubebeno & 1354 & 0,5 & 0,6 & - \\
\hline$\alpha$-copaeno & 1380 & 2,1 & 4,4 & - \\
\hline$\beta$-borboneno & 1388 & 0,7 & 2,8 & - \\
\hline$\beta$-elemeno & 1395 & 6,0 & 10,0 & - \\
\hline (E)-cariofileno & 1415 & 17,7 & 12,3 & - \\
\hline$\gamma$-elemeno & 1430 & 0,5 & - & - \\
\hline aromadendreno & 1437 & 0,3 & 0,1 & - \\
\hline$\alpha$-humuleno & 1450 & 1,3 & 0,3 & - \\
\hline allo-aromadendreno & 1457 & - & 1,1 & - \\
\hline germacreno-D & 1483 & - & 0,3 & 1,6 \\
\hline$\beta$-selineno & 1490 & 0,7 & - & - \\
\hline$\alpha$-muuroleno & 1503 & 0,6 & 0,7 & 1,1 \\
\hline$\beta$-bisaboleno & 1513 & 1,5 & 0,9 & - \\
\hline cis-calameneno & 1523 & 0,5 & - & - \\
\hline$\delta$ - cadineno & 1530 & - & 1,7 & - \\
\hline elemicina & 1557 & - & - & 24,5 \\
\hline trans-nerolidol & 1567 & 4,3 & 3,9 & - \\
\hline spatchulenol & 1580 & - & 5,3 & - \\
\hline óxido de cariofileno & 1584 & 32,1 & 26,6 & - \\
\hline óxido de humuleno & 1610 & 3,9 & - & - \\
\hline dilapiol & 1621 & - & - & 57,5 \\
\hline$\alpha$-muurolol & 1644 & 4,8 & - & - \\
\hline apiol & 1678 & - & - & 10,2 \\
\hline Sesquiterpenos & - & 77,5 & 71,0 & 2,7 \\
\hline Fenilpropanóides & - & - & - & 92,2 \\
\hline Total & - & 92,7 & 90,4 & 99,9 \\
\hline
\end{tabular}



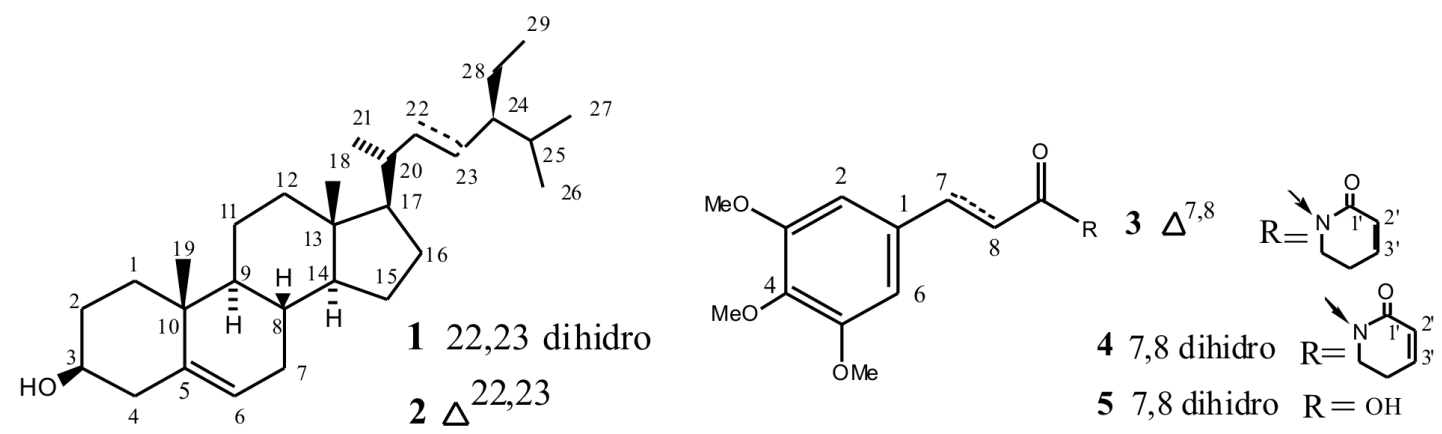

Figura 1 - Estruturas dos compostos 1 - 5, isoladas dos frutos de P. tuberculatum

Estudos fitoquímicos com folhas, sementes, talos e raízes de $P$. tuberculatum realizados nos estados do Amazonas, Ceará, Paraiba e São Paulo, revelaram a presença de 15 amidas (piplartina, dihidropiplartina, piplartina-dimerica A, pelitorina, piperlonguminina, dihidropiperlonguminina, piperina, dihidropiperina, piperina $S$, piperdardina, piperidina- $2 E, 4 E$-decadienamida, piperetina, $\mathrm{N}-(12,13,14-$ trimetoxidihidrocinnamoil)-delta(3)-piperidin-2-ona, cispiplartina, fagaramida), uma aristolactama (cefaranona B), e três derivados do acido cinâmico (ácido 3,4,5-trimetoxicinâmico, 6,7,8-trimetoxidihidrocinamato de metila e trans-6,7,8trimetoxicinamato de metila) (Braz-Filho et al., 1981; AraújoJúnior et al.,1997; Araújo et al., 1999; Navickiene et al., 2000; Cunha \& Chaves, 2001; Silva et al., 2002; Chaves et al., 2003; Miranda et al., 2003). Na Costa Rica e no Panamá, foram isoladas três amidas, duas comuns as encontradas nas espécies brasileiras, piplartine e dihydropiplartine e uma terceira, denominada piplaroxide (Capron \& Wiemer, 1996; Scout et al., 2005). Neste estudo com os frutos de P. tuberculatum, coletados no estado de Rondônia, foram identificados, pela primeira vez, o $\beta$-sitosterol 1 , o estigmasterol 2 e o ácido 3,4,5-trimetoxi-dihidrocinâmico 5. Com o isolamento de 5, é possível especular que o mesmo seja um precursor biossintético das amidas piplartina 3 e dihidropiplartina 4.

As determinações estruturais dos compostos 1-5 foram realizadas com base em dados de $\mathrm{RMN}$ de ${ }^{1} \mathrm{H}$ e ${ }^{13} \mathrm{C}$, uni e bidimensionais, espectros de massas e comparação com dados da literatura (Facundo et al., 2003; 2005b). Os dados de RMN ${ }^{1} \mathrm{H}$ e ${ }^{13} \mathrm{C}$ das substância 1 e 2 e 3-5, encomtram-se nas tabelas 2 e 3 , respectivamente.

O óleo essencial das raízes de $P$. hispidum, coletadas no estado de Rondônia, pertence ao quimiotipo dos fenilpropanóides e pode interessar as indústrias de cosméticos e inseticidas (Bergo et al., 2005). Além disto, este é o primeiro relato da composição química do óleo essencial das raízes dessa espécie e o perfil químico observado é diferente dos relatados para outras partes da planta (Machado et al., 1994; Santos et al., 2001; Pinto et al., 2004; Mesquita et al., 2005; Potzernheim et al., 2006).

Tabela 2 - Dados de RMN ${ }^{1} \mathrm{H}$ e ${ }^{13} \mathrm{C}$ dos compostos 1 e $\mathbf{2}\left(\mathrm{CDCl}_{3}\right)$.

\begin{tabular}{|c|c|c|c|c|c|c|c|c|c|}
\hline & & & & & & & & & 2 \\
\hline$C$ & $\delta_{c}$ & $\delta_{H}$ & $\delta_{\mathrm{c}}$ & $\delta_{\mathrm{H}}$ & $C$ & $\delta_{\mathrm{C}}$ & $\delta_{H}$ & $\delta_{\mathrm{c}}$ & $\delta_{H}$ \\
\hline 1 & 37,2 & - & 37,2 & - & 16 & 28,9 & - & 28,2 & - \\
\hline 2 & 29,7 & - & 29,7 & - & 17 & 56,1 & - & 56,9 & - \\
\hline 3 & 71,8 & $3,51(\mathrm{~m})$ & 71,8 & $3,51(\mathrm{~m})$ & 18 & 11,9 & $0,68(\mathrm{~s})$ & 12,1 & $0,69(\mathrm{~s})$ \\
\hline 4 & 39,8 & - & 39,6 & - & 19 & 18,9 & $1,02(\mathrm{~s})$ & 12,2 & $1,02(\mathrm{~s})$ \\
\hline 5 & 140,7 & - & 140,7 & - & 20 & 36,1 & - & 40,5 & - \\
\hline 6 & 121,7 & 5,36 (d) & 121,7 & 5,12 (d) & 21 & 18,8 & $0,93(\mathrm{~s})$ & 21,2 & $1,03(\mathrm{~s})$ \\
\hline 7 & 31,6 & - & 31,6 & & 22 & 33,7 & - & 138,3 & $5,22-4,95(\mathrm{~m})$ \\
\hline 8 & 31,9 & - & 31,9 & - & 23 & 26,1 & - & 129,3 & $5,22-4,95(\mathrm{~m})$ \\
\hline 9 & 50,1 & - & 50,1 & - & 24 & 45,8 & - & 50,1 & - \\
\hline 10 & 36,8 & - & 33,9 & - & 25 & 29,1 & - & 31,9 & - \\
\hline 11 & 21,1 & - & 21,1 & - & 26 & 19,4 & $0,83(\mathrm{~s})$ & 21,2 & $0,86(\mathrm{~s})$ \\
\hline 12 & 39,8 & - & 39,8 & - & 27 & 19,0 & $0,80(\mathrm{~s})$ & 19,8 & $0,81(\mathrm{~s})$ \\
\hline 13 & 42,3 & - & 42,3 & - & 28 & 23,1 & - & 25,4 & - \\
\hline 14 & 56,8 & - & 56,7 & - & 29 & 11,8 & $0,81(\mathrm{~s})$ & 11,9 & $0,83(\mathrm{~s})$ \\
\hline 15 & 24,4 & - & 24,3 & - & - & - & - & - & - \\
\hline
\end{tabular}


Tabela 3 - Dados de RMN ${ }^{1} \mathrm{H} \mathrm{e}^{13} \mathrm{C}$ dos compostos 3-5 $\left(\mathrm{CDCl}_{3}\right)$.

\begin{tabular}{ccccccc}
\hline \multicolumn{3}{c}{3} & \multicolumn{3}{c}{4} & \multicolumn{2}{c}{5} \\
\hline $\mathrm{C}$ & $\delta_{\mathrm{C}}$ & $\delta_{\mathrm{H}}$ & $\delta_{\mathrm{C}}$ & $\delta_{\mathrm{H}}$ & $\delta_{\mathrm{C}}$ & $\delta_{\mathrm{H}}$ \\
\hline 1 & 130,6 & - & 136,8 & - & 135,9 & - \\
2 & 105,4 & $6,81(\mathrm{~s})$ & 105,4 & $6,48(\mathrm{~s})$ & 105,2 & $6,43(\mathrm{~s})$ \\
\hline 3 & 153,3 & - & 153,0 & - & 153,2 & - \\
4 & 140,1 & - & 136,2 & - & 136,5 & - \\
5 & 153,3 & - & 153,0 & - & 153,2 & - \\
6 & 105,4 & $6,81(\mathrm{~s})$ & 105,4 & $6,48(\mathrm{~s})$ & 105,2 & $6,43(\mathrm{~s})$ \\
7 & 143,7 & $7,65(\mathrm{~d})$ & 24,5 & $3,25(\mathrm{t})$ & 35,7 & $2,90(\mathrm{t})$ \\
\hline 8 & 121,0 & $7,68(\mathrm{~d})$ & 40,8 & $2,93(\mathrm{t})$ & 31,0 & $2,68(\mathrm{t})$ \\
9 & 168,8 & - & 175,4 & - & 178,6 & - \\
$1^{\prime}$ & 165,8 & - & 165,3 & - & - & - \\
\hline $2^{\prime}$ & 125,8 & $6,04(\mathrm{dd})$ & 125,8 & $5,98(\mathrm{dd})$ & - & - \\
\hline $3^{\prime}$ & 145,5 & $6,93(\mathrm{~m})$ & 145,5 & $6,89(\mathrm{~m})$ & - & - \\
\hline $4^{\prime}$ & 24,7 & $2,48(\mathrm{~m})$ & 31,7 & $2,38(\mathrm{~m})$ & - & - \\
$5^{\prime}$ & 41,6 & $4,05(\mathrm{~m})$ & 40,9 & $3,97(\mathrm{t})$ & - & - \\
$\mathrm{OCH}_{3}-3$ & 56,6 & $3,88(\mathrm{~s})$ & 56,0 & $3,84(\mathrm{~s})$ & 56,7 & $3,84(\mathrm{~s})$ \\
\hline $\mathrm{OCH}_{3}-4$ & 61,3 & $3,86(\mathrm{~s})$ & 60,7 & $3,81(\mathrm{~s})$ & 60,8 & $3,83(\mathrm{~s})$ \\
\hline $\mathrm{OCH}_{3}-5$ & 56,6 & $3,88(\mathrm{~s})$ & 56,0 & $3,84(\mathrm{~s})$ & 56,7 & $3,84(\mathrm{~s})$ \\
\hline $\mathrm{OH}^{\prime}$ & - & - & - & - & - & $11,31(\mathrm{sl})$ \\
\hline
\end{tabular}

\section{BIBLIOGRAFIA CITADA}

Adams, R.P. 1995. Identification of essential oil components by gas chromatography/mass spectrometry. Allured Publ Corp., Carol Stream, IL, 469 pp.

Albiero, A.L.M., Paoli, A.A.S., Souza, L.A., Mourão, K.S.M. 2006. Morfoanatomia dos órgãos vegetativos de Piper hispidum Sw. (Piperaceae). Revista Brasileira de Farmacognosia, 16(3): 379391.

Araújo-Júnior, J.X., Cunha, V.L., Emidio, Chaves, M.C.0., GRAY, A.I. 1997. Piperdardine, a piperidine alkaloid from Piper tuberculatum. Phytochemistry, 44(3): 559-561.

Araújo-Júnior, J.X., Chaves, M.C.O., Cunha, E.V.L., Gray, A.I. 1999. Cepharanone B from Piper tuberculatum. Biochemical Systematics and Ecology, 27: 325-327.

Bergo, C.L., Mendonça, H. A., Silva, M.R. 2005. Efeito da época e freqüência de corte de pimenta longa (Piper hispidinervum $\mathrm{C}$. DC.) no rendimento de óleo essencial. Acta Amazonica, 35(2): 111-117.

Bezerra, D.P., Militão, G.C.G., Castro, F.O., Pessoa, C., Moraes, M.O., Silveira, E.R., Lima, M.A.S., Elmiro, M.J.F., CostaLotufo, L.V. 2007. Piplartine induces inhibition of leukemia cell proliferation triggering both apoptosis and necrosis pathways. Toxicology in Vitro, 21: 1-8.

Braz-Filho, R., Souza, M.P., Mattos, M.E.O. 1981. Piplartine-dimer A, a new alkaloid from Piper tuberculatum. Phytochemistry, 20(2), 345-6.

Capron, M.A., Wiemer, D.F. 1996. Piplaroxide, an ant-Repellent piperidine epoxide from Piper tuberculatum. Journal of Natural Products, 59(8): 794-795.
Chaves, M.C.O., Oliveira, A.H., Santos, B.V.O. 2006. Aristolactams from Piper marginatum Jacq. (Piperaceae). Biochemical Systematics and Ecology, 34: 75-77.

Chaves, M.C.O., Júnior, A.G.F., Santo, B.V.O. 2003. Amides from Piper tuberculatum fruits. Fitoterapia, 74: 181-183.

Cysne, J.B., Canuto, K.M., Pessoa, O.D.L., Nunes, E.P., Silveira, E.R. 2005. Leaf essential oils of four Piper species from the state of Ceará - northeast of Brazil. Journal of the Brazilian Chemical Society, 16(6B): 1378-1381.

Cunha, E,V.L., Chaves, M.C.O. 2001. Two amides from Piper tuberculatum fruits. Fitoterapia, 72: 197-199.

Facundo, V.A \& Moraes, S.M. 2003. Constituents of Piper aleyreanum (Piperaceae). Biochemical Systematics and Ecology, 31: 111-113.

Facundo, V.A \& Moraes, S.M. 2005a. Essential oil of Piper tuberculatum var. tuberculatum (Micq.) CDC leaves. Journal of Essential Oil Research, 17: 643-644.

Facundo, V.A., Silveira, A.S.P., Moraes, S.M. 2005b. Constituents of Piper alatabaccum Trel \& Yuncker (Piperaceae) Biochemical Systematics and Ecology, 31: 753-756.

Guimarães, E.F., Giordano, L.C.S. 2004. Piperaceae do nordeste brasileiro I: estado do Ceará. Rodriguésia, 55(84): 21-46.

Machado, M.F., Militão, J.S.L.T., Facundo, V.A., Morais, S.M., Machado, M.I.L. 1994. Leaf oils of two Brazilian Piper species: Piper arboreum Aublet var. latifolium (C.DC) Yuncker and Piper hispidum Sw. Journal of Essential Oil Research, 6, 643-644.

Maia, J.G.S., Zohbi, M.G.B., Andrade, E.H.A., Santos, A.S., Silva, M.H.L., Luz, A.I.R., Bastos, C.N. 1998. Constituents of the essential oil of Piper aduncum L. growing wild in the amazon region. Flavour and Fragrance Journal, 13(4): 269-272.

Mesquita, J.M.O., Cavaleiro, C., Cunha, A.P., Lombardi, J.A., Oliveira, A.B. 2005. Estudo comparativo dos óleos voláteis de algumas espécies de Piperaceae. Revista Brasileira de Farmacognosia, 15(1): 6-12.

Miranda, J.E., Navickiene, H.M.D., Couto, R.H.N., Bortoli, S.A., Kato, M.J., Bolzani, V.S., Furlan, M. 2003. Susceptibility of Apis mellifera (Hymenoptera:Apidae) to pellitorine, an amide isolated from Piper tuberculatum (Piperaceae). Apidologie, 34(4): 409-415.

Morais, S.M., Facundo, V.A., Bertini, L.M., Cavalcanti, E.S.B., Anjos-Jr, J.F., Ferreira, S.A., Brito, E.S., Souza, M.A. 2007. Chemical composition and larvicidal activity of essential oils from Piper species. Biochemical Systematics and Ecology, 35: 670-675.

Navickiene, H.M.D., Alécio, A.C., Kato, M.J., Bolzani, V.S., Young, M.C.M., Cavalheiro, A.J., Furlan, M. 2000. Antifungal amides from Piper hispidum and Piper tuberculatum. Phytochemistry, 55(6): 621-626.

Navickiene, H.M.D., Morandim, A.A., Alécio, A.C., Regasini, L.O., Bergamo, D.C.B., Telascrea, M., Cavaleiro, A.J., Lopes, M.N., Bolzani, V.S., Furlan, M., Marques, M.O.M., Young, M.C.M., Kato, M.J. 2006. Composition and antifungal activity of essential oils from Piper aduncum, Piper arboreum and Piper tuberculatum. Quimica Nova., 29(3); 467-470. 
Parmar, V.S., Jain, S.C., Bisht, K.S., Jain, R., Taneja, P., Jha, A., Tyagi, O.D., Prasad, A.K., Wengel, J., Olsen, C.E., Boll, P.M. 1997. Phytochemistry of the genus Piper. Phytochemistry, 46(4): 597-673.

Pino, J.A., Marbot, R., Bello, A., Urquiola, A. 2004. Composition of the essential oil of Piper hispidum Sw. from Cuba. Journal of Essential Oil Research, 16: 459-470.

Potzernheim, M., Bizzo, H.R., Costa, A.T.S., Vieira, R.F., Carvalho, C. M., Gracindo, L.A.M.B. 2006. Chemical characterization of seven Piper species (Piperaceae) from Federal District, Brazil, based on volatile oil constituents. Revista Brasileira de Plantas Medicinais, 8, 10-12.

Santos, P.R.D., Moreira, D.L., Guimarães, E.F., Kaplan, M.A.C. 2001. Essential oil of 10 Piperaceae species from the brazilian atlantic forest. Phytochemistry, 58(4): 547-551.
Scott, I.M., Puniani, E.H.J., Livesey, J.F., Poveda, L., Vindas, P.S., Durst, T., Arnason, J.T. 2005. Analysis of Piperaceae germplasm by HPLC and LCMS: A method for Isolating and Identifying unsaturated amides from Piper spp extracts. Journal of Agriculture and Food Chemistry, 53(6): 1907-1913.

Silva, R.V., Navickiene, H.M.D., Kato, M.J., Bolzani, V.S., Média, C.I., Young, M.C.M., Furlan, M. 2002. Antifungal amides from Piper arboreum and Piper tuberculatum. Phytochemistry, 59(5): 521-527.

Silva, W.C., Ribeiro, J.D’Arc, Souza, H.E.M., Corrêa, R.S. 2007. Atividade inseticida de Piper aduncum L. (Piperaceae) sobre Aetalion sp. (Hemiptera: Aetalionidae), praga de importância econômica no Amazonas. Acta Amazonica, 37(2): 293-298.

Recebido em 06/06/2008

Aceito em 28/08/2008 